TRANS $\cdot$ núm. $24 \cdot 2020$

MISCELÁNEA $\cdot 205-228$
Con el fin de destacar el aporte de la línea de apoyo a la traducción del Ministerio de las Culturas, las Artes y el Patrimonio del gobierno de Chile, en este artículo se describen los 90 proyectos de traducción financiados en el período 2013 a 2017 por este concurso y se reflexiona sobre su contribución a la institucionalización de la disciplina y de la práctica de la traducción en nuestro país. Entre los resultados se destacan el aumento anual sostenido del número de proyectos y de los fondos asignados, la baja participación de responsables residentes en regiones del norte y sur del país y la alta presencia de traducciones de géneros literarios, como poesía, novela y ensayo. Finalmente, se proponen algunas medidas para continuar optimizando el proceso de institucionalización de la traducción en nuestro país.

PALABRAS CLAVE: apoyo a la traducción, institucionalización de la disciplina, práctica de la traducción, fomento del libro y la lectura.

\title{
La línea de apoyo a la traducción del Consejo Nacional del Libro y la Lectura. Un aporte a la institucionalización de la traducción en Chile
}

María IsABel DiéGuez Morales

Pontificia Universidad Católica de Chile

Javiera Paz Sepúlveda Salas

Traductora independiente

\section{The Translation Support Fund of the Consejo Nacional del Libro y la Lectura: a contribution to the institutionalization of translation in Chile}

In order to highlight the contribution of the translation support fund administered by the Ministerio de las Culturas, las Artes y el Patrimonio of the Chilean administration, in this article we aim at describing the 90 translation projects approved by this fund in the period from 2013 to 2017, and at reflecting on its contribution to the institutionalization of the discipline and practice of translation in our country. Among the results are the sustained annual increase in the number of projects and the funds allocated to them, the low participation of translators that reside in the north and south of the country, and the high presence of translations of literary genres, such as poetry, novels and essays. Finally, some measures that contribute to the optimization of the institutionalization of translation in our country will be proposed.

KEY WORDS: translation support, institutionalization of the discipline, practice of translation, book and reading promotion. 


\section{INTRODUCCIÓN}

El año 2013, el Ministerio de las Culturas, las Artes y el Patrimonio del gobierno de Chile abrió las postulaciones al concurso público de apoyo a la traducción el cual es administrado por el Consejo Nacional del Libro y la Lectura, entidad que asigna los recursos del Fondo Nacional de Fomento del Libro y la Lectura, en adelante, el Fondo. Su objetivo es «fomentar y promover proyectos, programas y acciones de apoyo a la creación literaria, la promoción de la lectura, la industria del libro, la difusión de la actividad literaria, el fortalecimiento de las bibliotecas públicas y la internacionalización del libro chileno» (Consejo Nacional del Libro y la Lectura, 2019).

Este Fondo está dividido en cinco áreas: Fondart Nacional, Fondart Regional, Fondo Audiovisual, Fondo de la Música, y Fondo del Libro y la Lectura. Este último cuenta con una serie de líneas de concurso entre las que se encuentra, desde el año 2013, la línea de apoyo a la traducción, dirigida a traductores y profesionales del libro, cuyo objetivo es fomentar las traducciones en nuestro país, dando respuesta a lo establecido en la Política Nacional de la Lectura y el Libro 2015-2020 (PNLL) promulgada por el gobierno de Chile el año 2015 ${ }^{1}$. Esta política gubernamental surgió del trabajo, entre agosto y octubre de 2014, de mesas público-privadas integradas por más de 700 personas entre las que se destacaban autores, editores, libreros, profesores, traductores y académicos. También colaboraron en esta iniciativa representantes del Colegio de Traductores e Intérpretes de Chile (COTICH), a través de su Comisión de Industria Editorial. Sus represen-

I Brodsky (2013) ofrece un panorama del espacio editorial chileno y una breve historia de la constitución en 2001 de la Mesa del Libro, antecedente directo de la Política Nacional del Libro y la Lectura, promulgada en su primera versión el año 2006. tantes participaron en la mesa de trabajo «Industria e Internacionalización» junto a impresores, editores, libreros, representantes de la Corporación de Fomento de la Producción (CORFO) y PROCHILE, entidad que promueve las exportaciones de productos y servicios del país, entre otros.

El objetivo general que se propuso el gobierno al promulgar esta política fue:

crear las condiciones para asegurar a todos los habitantes del país, incluyendo a los pueblos originarios con sus lenguas y a las comunidades tradicionales, rurales y de inmigrantes, la participación y el acceso a la lectura, el libro, la creación, el patrimonio y los saberes, protegiendo y fomentando la diversidad cultural y territorial, con equidad e integración social (Política Nacional del Libro y la Lectura, 2015: 26).

El documento se articula en cinco ámbitos: Lectura, Creación, Patrimonio bibliográfico, Industria e internacionalización, y Marco jurídico institucional. Cada uno de estos ámbitos establece un propósito orientador, objetivos, medidas y designa la(s) institución(es) responsable(s) de implementar dichas medidas y, con ello, cumplir los objetivos propuestos.

El propósito orientador del primer ámbito de la PNLL concibe la lectura como parte fundamental de los derechos económicos, sociales y culturales de las personas y la considera esencial en la formación de personas creativas, reflexivas, críticas y participativas. Con este fin, la Política determina, entre otros objetivos, «garantizar que la lectura y el acceso al libro sean utilizados como fuente de información y aprendizaje, y para la integración de los pueblos indígenas y de las personas con capacidades diferentes»y, para poder cumplirlo, destaca como una de sus medidas «promover la traducción desde y hacia las lenguas de los pueblos originarios» (Política Nacional del Libro y la Lectura, 2015: 30). 
El cuarto ámbito, Industria e internacionalización, pone de relieve la importancia de desarrollar una industria del libro nacional y regional sostenible con el fin de aumentar la producción creativa e intelectual. Para ello, se enuncian tres medidas relacionadas directamente con la traducción. La primera medida corresponde a «traducir a lenguas originarias obras patrimoniales de los pueblos indígenas, editadas en sus primeras versiones en otros idiomas», la cual permite cumplir el objetivo de «fomentar la creación y el emprendimiento editorial de los pueblos indígenas» (Política Nacional del Libro y la Lectura, 2015: 36). Las otras dos medidas de este cuarto ámbito corresponden a «fortalecer y perfeccionar el programa de traducciones de autores chilenos a otros idiomas (...) y contribuir al fortalecimiento de la industria editorial nacional y su internacionalización mediante la traducción en Chile de autores de otras lenguas al español». Ambas medidas contribuyen al objetivo de «apoyar y promover la internacionalización de la industria editorial nacional y regional» (Política Nacional del Libro y la Lectura, 2015: 37).

En sus primeros cinco años de ejecución, desde la primera convocatoria en 2013 y hasta la convocatoria 2017, el Fondo ha financiado 90 proyectos de traducción mediante la entrega de apoyo total o parcial para la traducción al castellano de obras escritas por autores y autoras extranjeros, y para la traducción de autores y autoras nacionales a lenguas extranjeras. Las obras por traducir pueden ser de ficción o no ficción y pertenecer a cualquier género; además, deben estar editadas en formato digital o impreso y se debe asegurar su futura distribución y comercialización en nuestro país mediante una carta de compromiso de publicación por parte de una casa editorial. Además de este requisito, el responsable de la postulación debe adjuntar la autorización o cesión de derechos de autor de la obra a traducir. Este programa financia la labor del traductor o traductora y de equipos de profesionales, tales como editores, correctores de prueba y estilo, entre otros. Cada proyecto recibe un apoyo de un máximo de $\$ 4$ millones de pesos chilenos para gastos de honorarios y de operación, referidos a servicios de traducción, corrección de prueba, corrección de estilo y edición. Además, los proyectos deben iniciarse durante el año de su adjudicación y no pueden superar los 18 meses de duración.

Las postulaciones se abren cada año por un período de veinte días hábiles desde la publicación de las bases del concurso y se puede enviar la postulación digital a través del sitio web del Fondo, o bien en formato impreso por correo certificado. Una vez que se constata el cumplimiento de las bases, se declara la admisibilidad del proyecto el cual se somete posteriormente a una evaluación y selección por parte de una Comisión de Asesores del Fondo quienes emplean una pauta de evaluación con criterios de calidad como la relevancia de la obra y autor a traducir, la coherencia del proyecto, el currículum del traductor y de la editorial que publicará la obra, como también el presupuesto solicitado.

Cabe destacar que, además de la realización de la traducción, los responsables se comprometen a realizar una actividad de difusión del proyecto de traducción en establecimientos escolares de educación pública y en las comunidades próximas a ellos, en conformidad con lo que establece la respectiva Ley de Presupuesto del gobierno. Dicha actividad no se puede financiar con recursos del Fondo y se puede llevar a cabo en el marco de los programas de Educación Artística que ejecuta el Ministerio de las Culturas, las Artes y el Patrimonio (Bases del concurso público del programa de apoyo a la traducción, 2019: 4).

Con el fin de poner de relieve esta política pública de fomento a la traducción, inédita en 
208 nuestro país, nos hemos propuesto como objetivos caracterizar el proceso de institucionalización de la disciplina y de la práctica de la traducción en Chile, describir las principales características de los 90 proyectos de traducción financiados en sus primeros 5 años por la línea de apoyo a la traducción y proponer algunas medidas que permitan optimizar el proceso de institucionalización de la disciplina y de la práctica de la traducción en nuestro país.

\section{INSTITUCIONALIZACIÓN DE LA DISCIPLINA Y DE LA PRÁCTICA DE LA TRADUCCIÓN EN CHILE}

Para abordar este tema a nivel nacional, describiremos primero algunos rasgos relevantes del proceso de institucionalización de la disciplina y de la práctica de la traducción a nivel global puesto que este proceso ha tenido una clara incidencia en su evolución en Chile. Lambert (2013) destaca que, a pesar de tratarse de una práctica antiquísima, la profesión tanto de traductores como intérpretes sólo comenzó paulatinamente a recibir un reconocimiento a mediados del siglo xx cuando, luego de la Segunda Guerra Mundial, se iniciaron los servicios profesionales multilingües, lo cual dio paso a la creación de carreras universitarias para formar traductores e intérpretes profesionales. De esta forma, se inició gradualmente el proceso de institucionalización tanto de la práctica como de la disciplina de la traducción y la interpretación. A su vez, algunas empresas, como IBM, Siemens y, más tarde, organizaciones como la Unión Europea, iniciaron en las décadas siguientes ambiciosos proyectos sobre la entonces novedosa área de traducción automática (Lambert, 2013: 7-9). Este autor señala que uno de los hechos más espectaculares en el área de las humanidades fue el establecimiento gradual de la disciplina de Estudios de la traducción en al menos 150 universidades en los cinco continentes. En el año 1947 se crea la Federación Internacional de Traductores (FIT) y las primeras generaciones de traductores profesionales comienzan a impulsar iniciativas como la organización de simposios y seminarios, y la creación de revistas y asociaciones gremiales con el fin de compartir su experiencia profesional e intelectual (Lambert, 2013: 10-11) ${ }^{2}$.

Sin embargo, esos fueron solo los primeros pasos de un largo proceso de institucionalización de la disciplina y la práctica de la traducción que, no cabe duda, adquirió nuevos bríos con el proceso de globalización que se inició en los años 70. Cronin (2013: 491) destaca que la globalización sería prácticamente impensable sin traducción ni traductores, dada la naturaleza multilingüe de la comunidad mundial. Prueba de lo anterior es la alta demanda de traducción en organismos internacionales como Naciones Unidas, y el Banco Mundial, y en organizaciones no gubernamentales como Amnistía Internacional y Médicos sin Fronteras, por nombrar algunos ${ }^{3}$.

En nuestro país, los primeros pasos del proceso de institucionalización de la disciplina se inician de manera sistemática a comienzos de la década de los 70 del siglo XX, si bien la traducción se practicaba en diferentes ámbitos del quehacer nacional ya a principios del siglo anterior, cuando, en 1812, se crea La Aurora de Chile, primer periódico a cargo de Camilo Henríquez quien «difundió en español las ideas de El Contrato Social de Rousseau» (Cabrera, 1995: 1317), hecho que demuestra la incidencia que tuvo la traducción en el desarrollo político, social y cultural del país durante todo el siglo XIX. Digno

\footnotetext{
2 Para una descripción detallada del proceso de institucionalización de la traducción a nivel mundial, véase Lambert (2013).

3 Cronin (2013) presenta una síntesis detallada y actualizada de la relación entre traducción y globalización.
} 
también de destacar es la Biblioteca Chilena de Traductores (1820-1924) de José Toribio Medina (1925) y, especialmente, su segunda edición aumentada y corregida donde Payàs (2007) presenta un acucioso estudio preliminar en el cual destaca, entre muchos otros aspectos, las numerosas traducciones de obras pedagógicas realizadas entre los años 1840 y 1890 , hecho que demuestra el «espíritu educativo e instrumental de la traducción» (Payàs, 2007: 48).

En el ámbito académico, dos universidades inician en 1971 y 1972 sus programas de estudio en traducción de inglés, alemán y francés al español, la Pontificia Universidad Católica de Chile y la Universidad de Concepción, respectivamente. Una década después, otras universidades comienzan a ofrecer la carrera de traducción y (o) interpretación y a la fecha se cuenta con un total de 13 instituciones de educación superior que ofrecen el grado de licenciatura en traducción (Basaure y Contreras, 2019: 167) ${ }^{4}$. Al alero de algunas de estas instituciones, se inicia a partir de la década de los 80 un trabajo incipiente en el ámbito de la investigación en traducción, en un inicio con el apoyo interno de las propias universidades y, más tarde, con fondos externos ofrecidos por la Comisión Nacional de Investigación Científica y Tecnológica (CONICYT), principalmente a través de su programa Fondo Nacional de Desarrollo Científico y Tecnológico $\left(\right.$ FONDECYT) ${ }^{5}$.

4 Basaure y Contreras (2019) presentan una lista actualizada de las universidades e institutos profesionales que ofrecen la carrera de traducción a nivel de pregrado y posgrado en nuestro país.

5 La base de datos de proyectos FONDECYT Regular adjudicados en los últimos 25 años (1993-2018) en el área de Filología y Lingüística consta de 126 proyectos de los cuales solo un $10 \%$ corresponde a investigaciones relacionadas con el área de traducción, específicamente traducción filológica, traducción automática, mediación mapudungun-castellano y terminología. En la base de datos de proyectos FONDECYT
En efecto, a dos décadas de creada la carrera 209 de traducción, un equipo de investigadores del Programa de Traducción de la Pontificia Universidad Católica de Chile realiza un diagnóstico sobre el desarrollo de la disciplina de la traducción en Argentina, Chile y Perú en el cual afirman que en su estudio «quedó de manifiesto el incipiente estado de desarrollo de la investigación en el área de la traducción» en los países estudiados (Cabrera et al., 1991: 5).

Dignas de destacar son también algunas iniciativas de vinculación con el medio organizadas por diferentes universidades como las Jornadas Nacionales de Enseñanza de la Traducción y la Interpretación que al año 2018 sumaban siete versiones y las cuatro Jornadas de Investigación en Traducción organizadas por el programa de Magíster en Traducción de la Pontificia Universidad Católica de Chile, amén de un sinnúmero de cursos y talleres de perfeccionamiento organizados por diferentes universidades, los cuales permiten a los traductores profesionales mantenerse actualizados en diferentes materias, como por ejemplo, teorías traductológicas modernas, tecnologías aplicadas a la traducción, gestión terminológica, y revisión y control de calidad de la traducción, entre otras.

En el ámbito gremial, en el año 1991 se crea el Colegio de Traductores e Intérpretes de Chile, COTICH, miembro de la FIT. Su principal objetivo es «proteger los intereses y regularizar el ejercicio de la profesión, impulsar el perfeccionamiento de sus asociados y contribuir en forma efectiva al desarrollo cultural, económico y social del país» (Colegio de Traductores e Intérpretes de Chile, 2019). Entre sus numerosas activida-

de Inicio, el porcentaje es aún menor (8\%), dado que desde 2007, año en que comienza este concurso, hasta el 2018 se realizaron 62 proyectos y solo 5 están relacionados con la traducción en áreas como la traducción teatral, terminología, interpretación y traducción filológica. 
210 des, se destacan las cuatro versiones del Congreso de Traducción e Interpretación celebrados los años 2011, 2012, 2014 y 2017, y el patrocinio del Premio de Traducción a la Excelencia Profesional y Académica que confiere anualmente desde 1995 el Programa de Traducción de la Pontificia Universidad Católica de Chile a destacados traductores del ámbito nacional, una de las iniciativas que plasma la colaboración estrecha que existe entre los ámbitos gremial y académico.

Por otra parte, con el fin de caracterizar el mercado de la traducción en Chile, se han llevado a cabo investigaciones como las que ha realizado el Programa de Traducción de la Pontificia Universidad Católica de Chile los años 2006, 2008, 2011 y 2015 sobre las características del trabajo que efectúan los traductores profesionales residentes en Chile (Diéguez et al., 2014, 2015 y 2016); el estudio sobre el nivel de éxito laboral subjetivo de traductores chilenos (Delgado, 2017); la encuesta a agencias de traducción de Santiago de Chile llevada a cabo por Araya (2013) en su tesis sobre el enfoque por competencias en la formación de traductores, y la encuesta a profesores de traducción en el marco del estudio sobre la subcompetencia instrumental profesional en el currículo de traducción en Chile (Micheli, 2013).

En el ámbito jurídico, se destacan, entre otros documentos, el Código de Procedimiento Civil (1902/2019) que regula el papel del perito traductor/intérprete en actuaciones judiciales y la traducción de documentos extendidos en lengua extranjera, y la Ley de Propiedad Intelectual (1970/2017), instrumento que reconoce al traductor como sujeto del derecho de autor de la obra derivada de la obra originaria.

En el ámbito normativo, existen dos normas chilenas promulgadas por el Instituto Nacional de Normalización (INN) las cuales regulan la práctica de la traducción. La norma NCh 3124.
Of2008 Servicios de traducción - Requisitos para la prestación de servicios está basada en la versión oficial en inglés de la norma europea BS-EN 15038: 2006 Translation services - Service requirements y su objetivo es «establecer y definir los requisitos para que los proveedores de servicios de traducción proporcionen servicios de calidad» (NCh 3124.Of2008, 2008:1). Por otra parte, la norma NCh 3159.0f2008 Documentación - Presentación de traducciones corresponde a «una traducción idéntica de la versión en inglés de la norma internacional ISO 2384:1977 Documentation - Presentation of translations» (NCh 3159.of2008, 2008: 1). En esta segunda norma se establecen reglas para asegurar la normalización de las traducciones, con el fin de simplificar su uso por parte de diferentes usuarios.

Luego de esta somera descripción de los hitos más relevantes que han contribuido a la institucionalización de la disciplina y de la práctica de la traducción en nuestro país, nos abocaremos, como ya señalamos anteriormente, a describir las principales características de los 90 proyectos de traducción financiados en el período 2013-2017 por el Fondo de Fomento del Libro y la Lectura.

\section{MÉTODO}

Con el fin de cumplir los objetivos propuestos, hemos realizado una investigación de enfoque mixto dado que se analizan datos cuantitativos a partir de una base de datos proporcionada por la Subsecretaría de las Culturas y las Artes del gobierno de Chile. Los datos se complementan con un análisis de los resultados de tipo cualitativo que dice relación con el aporte a la institucionalización de la disciplina y la práctica de la traducción en Chile del concurso de apoyo a la traducción. Por tratarse del primer estudio sobre este concurso, la investigación tiene un 
alcance exploratorio-descriptivo y un diseño no experimental en el cual se utilizó la observación y el análisis de contenido como instrumentos de recolección y análisis de los datos.

Para obtener el corpus de trabajo, las investigadoras elevaron a fines de octubre de 2018 una solicitud al Consejo para la Trasparencia del gobierno de Chile con el fin de obtener la base de datos de los proyectos de la línea de apoyo a la traducción aprobados por el Fondo en el período 2013-2017. En la solicitud se especificaban las razones y los objetivos de la investigación que se pretendía realizar. A comienzos de noviembre, la Subsecretaría de las Culturas y las Artes envió la base de datos de los 90 proyectos ejecutados entre 2013 y 2017 y una carta en donde se informaba que se había excluido de la base de datos información sobre correos electrónicos, números de teléfono y direcciones personales de los responsables de los proyectos en atención a la protección de datos personales, citando las leyes que cautelan dicha información. Una metodología similar se empleó para obtener la base de datos (1993-2018) de los proyectos FONDECYT Regular y de Inicio del área de Filología y Lingüística, solicitud que fue realizada a través del sitio del Consejo para la Trasparencia, quienes derivaron a su vez dicho requerimiento a CONICYT.

El corpus de trabajo de los proyectos de apoyo a la traducción corresponde una base de datos en la cual se presentan los siguientes campos de información sobre cada proyecto: folio, fecha de recepción, nombre del proyecto, responsable, tipo de persona (natural o jurídica), modalidad (autores chilenos a lenguas extranjeras, o bien, autores extranjeros al castellano), obra a traducir, autor(a), formato (impreso o digital), idioma a traducir, región de residencia, monto solicitado, monto adjudicado, situación (seleccionado) y puntaje obtenido.
En el caso de la base de datos de los proyectos FONDECYT del área de Filología y Lingüística del período 1993-2018, los campos de información son los siguientes: folio del proyecto, título, instrumento (proyecto regular o de inicio), año del concurso, nombre del investigador o investigadora, calidad, es decir, responsable o coinvestigador(a), grupo de estudio, disciplina, institución patrocinante, fechas de inicio y término del proyecto y sector de aplicación. Esta última base de datos permitió elaborar la nota 5 que describe el porcentaje de proyectos relacionados con traducción que ha aprobado FONDECYT en los últimos 25 años.

Las características de los proyectos que se presentarán a continuación se han extraído de la base de datos de los proyectos de apoyo a la traducción descrita más arriba y corresponden a: número de proyectos y montos aprobados para su ejecución; tipos de personas responsables, es decir, naturales, jurídicas o extranjeras; región de residencia de los responsables de los proyectos; tipo de traducciones, vale decir, autotraducciones (traducción de una obra realizada por su propio autor), o bien traducciones realizadas por traductores diferentes del autor o autora; pares de lengua de traducción; tipo de formato, sea este impreso o digital; tipos de texto, y géneros de los textos traducidos.

\section{RESULTADOS}

A continuación, se presentan los resultados obtenidos luego de analizar la base de datos de los 90 proyectos de traducción financiados por el Fondo entre 2013 y 2017.

\section{Número de proyectos y fondos adjudicados}

En cuanto al número de proyectos de traducción adjudicados anualmente, en la Tabla 1 se 
212 observa un aumento sostenido en el período de cinco años, en especial en los últimos dos años; cabe destacar que el número de proyectos del año 2017 prácticamente triplica el número de proyectos del primer año de apertura del concurso. De igual forma, en la Tabla 1 se observa un aumento considerable en el monto de dinero en pesos chilenos asignado cada año para el financiamiento de los proyectos. Al comparar los fondos del año 2013 y del 2017, se observa que el último es ocho veces mayor que el primero.

Tabla 1. Número de proyectos y fondos adjudicados por año

\begin{tabular}{ccc}
\hline Año & $\begin{array}{c}\text { Número } \\
\text { de proyectos }\end{array}$ & $\begin{array}{c}\text { Fondos adjudicados por } \\
\text { año (CLP) }\end{array}$ \\
\hline 2013 & 10 & $17.234 .348 .-$ \\
\hline 2014 & 12 & $40.701 .433 .-$ \\
\hline 2015 & 15 & $39.661 .666 .-$ \\
\hline 2016 & 24 & $60.424 .385 .-$ \\
\hline 2017 & 29 & $135.224 .507 .-$ \\
\hline Total: & 90 & $\mathbf{2 9 3 . 2 4 6 . 3 3 9 . -}$ \\
\hline
\end{tabular}

\section{Tipos de personas responsables de los proyectos}

Como se puede observar en la Tabla 2, la mayoría de las personas responsables de los 90 proyectos adjudicados en el período corresponden a personas naturales $(74,4 \%)$, frente a personas jurídicas $(25,5 \%)$, si bien se observa un paulatino aumento de estas últimas a partir del año 2014. En el primer año del concurso (2013), solo participaron personas naturales como responsables de los proyectos; sin embargo, en el último año del concurso (2017) la participación de personas jurídicas ascendió a un $45 \%$ frente a las personas naturales.
Tabla 2. Tipo de personas responsables de los proyectos

\begin{tabular}{ccc}
\hline Año & Persona jurídica & Persona natural \\
\hline 2013 & 0 & 10 \\
\hline 2014 & 3 & 9 \\
\hline 2015 & 6 & 9 \\
\hline 2016 & 5 & 19 \\
\hline 2017 & 9 & 20 \\
\hline Total & $\mathbf{2 3}(\mathbf{2 5 , 5 \% )}$ & $\mathbf{6 7 ( 7 4 , 4 \% )}$ \\
\hline
\end{tabular}

En la tabla 3 podemos ver que la participación de personas naturales extranjeras con residencia en nuestro país corresponde a casi un $21 \%$ del total de 67 personas naturales responsables de los proyectos. Si bien este porcentaje es similar al que presentan las personas jurídicas frente a las personas naturales (cf. tabla 2), los datos muestran que su participación ha ido disminuyendo en el período, de un 50\% el primer año de la convocatoria a solo un 5\% el año 2017.

Tabla 3. Tipo de persona natural responsable de los proyectos

\begin{tabular}{ccc}
\hline Año & $\begin{array}{c}\text { Persona natural } \\
\text { chilena }\end{array}$ & $\begin{array}{c}\text { Personal natural } \\
\text { extranjera }\end{array}$ \\
\hline 2013 & 5 & 5 \\
\hline 2014 & 7 & 2 \\
\hline 2015 & 7 & 2 \\
\hline 2016 & 15 & 4 \\
\hline 2017 & 19 & 1 \\
\hline Total & $\mathbf{5 3}(\mathbf{7 9 , 1 \% )}$ & $\mathbf{1 4}(\mathbf{2 0 , 9 \% )}$ \\
\hline
\end{tabular}

Residencia de los responsables de los proyectos

Para analizar los datos de la región de residencia de los responsables de los proyectos de traduc- 


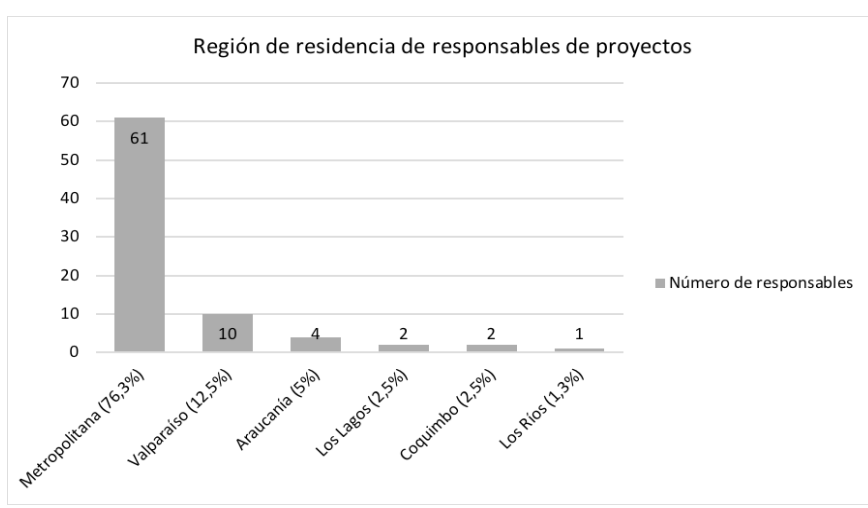

GRÁFICO I. Región de residencia de los responsables de los proyectos

ción no se consideraron 10 respuestas que solo indicaban en la base de datos la palabra «extranjero», sin especificar la región de residencia. Como se puede observar en el gráfico 1, la mayor parte de los responsables de los proyectos residen en la Región Metropolitana (76,3\%), seguidos por aquellos que viven en la quinta región de Valparaíso con un $12,5 \%$ y en la novena región de la Araucanía con un 5\%. Las regiones de Los Lagos y Coquimbo presentan porcentajes muy bajos (2,5\%), al igual que la región de Los Ríos con un $1,3 \%$.

Las cifras desglosadas por años que aparecen en el gráfico 2 demuestran un aumento incipiente de los responsables de proyectos residentes en regiones del sur y norte del país. Se observa que en el año 2013 no hubo presencia de responsables de proyectos residentes en regiones. Su participación comenzó el año 2014 con un 10\% y luego fue aumentando en los años siguientes hasta alcanzar un promedio de un $28 \%$ entre los años 2015 y 2017.

\section{Tipo de traducción realizada}

Por otra parte, un alto porcentaje $(84,4 \%)$, es decir 76 proyectos, corresponde a traducciones realizadas por una persona diferente del autor de los textos originales y solo un $15,5 \%$ a autotraducciones (14), es decir, proyectos en los cuales los propios autores de los textos originales traducen sus obras a otras lenguas ${ }^{6}$.

\section{Lenguas de traducción}

En la tabla 4 se presentan los pares de lengua de traducción distribuidos en el período de cinco años. Se contabilizaron un total de 19 pares de lengua diferentes entre los cuales el más frecuente es del «inglés al español» con 25 proyectos, seguido de 17 proyectos del «español al francés», 14 del «español al inglés»y 9 del «francés al español». A continuación, se presentan 5 proyectos del «alemán al español», 4 del «español al italiano» y 3 del «italiano al

6 Para una descripción de la evolución histórica y una detallada clasificación de la autotraducción, véase Recuenco Peñalver (2011). 


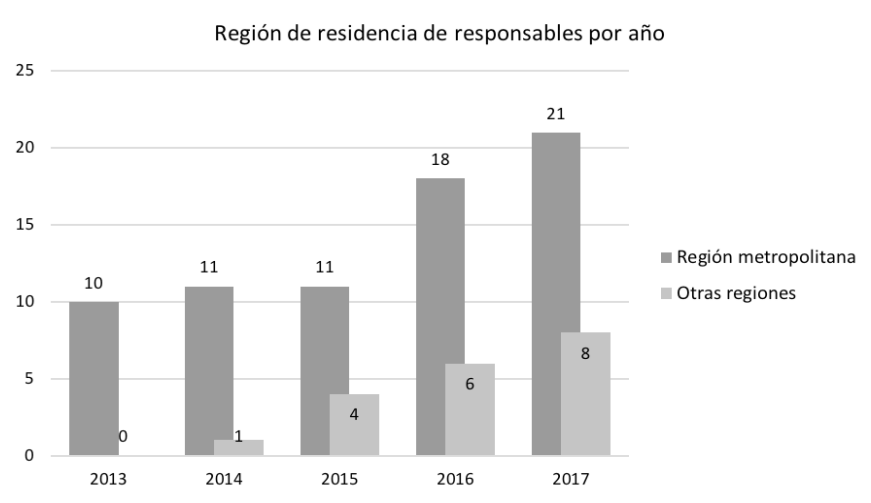

GRÁFICO 2. Región de residencia de los responsables por año

español». Los pares de lengua del «español al croata», «español al alemán», «griego al español»y «portugués al español» presentan una frecuencia de 2 proyectos cada uno. Finalmente, ocho pares de lengua presentan la frecuencia mínima con un proyecto cada uno: «español al portugués», «español al griego», «español al chino», «tibetano al español», «español al polaco», «sueco al español», «latín al español»y «ruso al español».

\section{Formato de las traducciones}

En cuanto al formato de las traducciones, un elevado porcentaje $(95,5 \%)$ se presenta como texto impreso y solo un $5,5 \%$ en formato digital. Cabe destacar que en los primeros dos años del concurso, 2013 y 2014, se presentan solo 4 de los 22 proyectos en formato digital y en el año 2016 una traducción se presenta en ambos formatos. En las convocatorias de los años 2015 y 2017 ningún proyecto se presenta en formato digital.

\section{Tipo de texto y género}

En lo que respecta al tipo de texto que se traduce, un elevado porcentaje corresponde a textos literarios $(74,4 \%)$, frente a textos no literarios $(25,5 \%)$; estos últimos corresponden en su mayoría a monografías, es decir, estudios sobre un área temática específica. A su vez, los géneros más frecuentes en los textos literarios corresponden a poesía $(43,3 \%)$, novela $(29,8 \%)$ y ensayo (11,9\%). La literatura de viajes representa solo un $4,5 \%$ y los géneros menos frecuentes corresponden a aforismos, drama y cuento con un $3 \%$ cada uno. Solo se presenta una autobiografía que representa un 1,5\% del total de textos literarios (cf. Gráfico 3).

En cuanto a los temas que se abordan en las monografías que corresponden al género no literario, estas presentan una amplia variedad, destacándose, entre otros, la filosofía, política nacional e internacional, sociología, e historia y cultura de la Isla de Pascua. También se abordan temas como pueblos originarios de Chile, cinematografía, arte e historia del arte, medio ambiente, 
Tabla 4. Pares de idiomas de traducción

\begin{tabular}{|c|c|c|c|c|c|c|}
\hline Pares de lenguas & 2013 & 2014 & 2015 & 2016 & 2017 & TOTAL \\
\hline Inglés al español & & & 5 & 7 & 13 & 25 \\
\hline Español al francés & 3 & 5 & & 3 & 6 & 17 \\
\hline Español al inglés & 3 & 4 & 2 & 5 & & 14 \\
\hline Francés al español & & & 4 & 5 & & 9 \\
\hline Alemán al español & & & 1 & 1 & 3 & 5 \\
\hline Español al italiano & 1 & 2 & & 1 & & 4 \\
\hline Italiano al español & & & & & 3 & 3 \\
\hline Español al croata & 2 & & & & & 2 \\
\hline Español al alemán & & 1 & & 1 & & 2 \\
\hline Portugués al español & & & & & 2 & 2 \\
\hline Griego al español & & & 1 & & 1 & 2 \\
\hline Español al griego & & 1 & & & & 1 \\
\hline Español al portugués & 1 & & & & & 1 \\
\hline Español al chino & & & 1 & & & 1 \\
\hline Tibetano al español & & & 1 & & & 1 \\
\hline Español al polaco & & & & 1 & & 1 \\
\hline Sueco al español & & & & 1 & & 1 \\
\hline Latín al español & & & & 1 & & 1 \\
\hline Ruso al español & & & & & 1 & 1 \\
\hline Total /año & 10 & 13 & 15 & 26 & 29 & 93 \\
\hline
\end{tabular}

teoría queer, didáctica de la traducción, medios sociales, urbanismo y pensamiento mítico.

\section{Otros datos relevantes a partir del cruce de variables}

Al cruzar algunas variables de la base de datos, se observa en primer lugar que en el caso de los 23 proyectos presentados por personas jurídicas, solo una de ellas tiene su residencia en la región de Valparaíso y el resto reside en la Región Metro- politana. Los pares de lengua de mayor frecuencia de traducción de estos proyectos son del «inglés al español» (10) y del «francés al español» (6), lo cual coincide con los resultados generales del estudio dado que estos pares de lengua se ubican entre los primeros cuatro de mayor frecuencia de traducción. Por último, el tipo de género textual que se traduce corresponde principalmente a textos literarios (61\%), en comparación con la traducción de monografías (39\%).

En segundo lugar, en el caso de las personas 


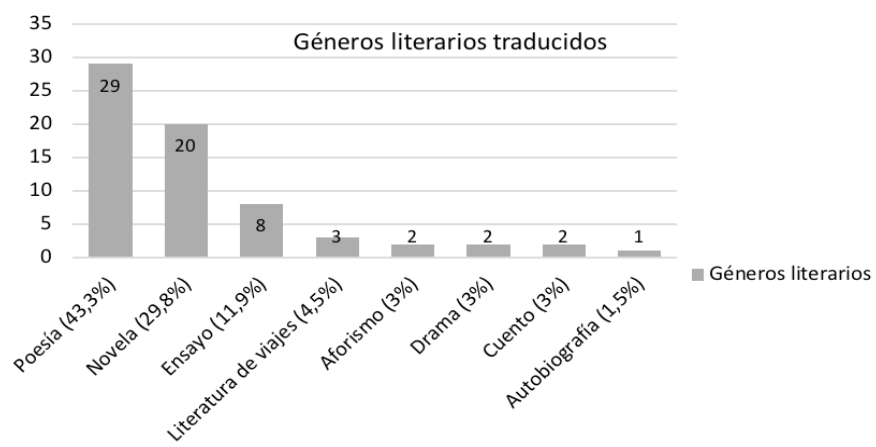

GRÁFICO 3. Géneros literarios traducidos

naturales extranjeras con residencia en nuestro país que son responsables de 14 proyectos, se observa que más de un $60 \%$ de ellas traduce del español a lenguas extranjeras, principalmente al inglés y al francés. Al igual que en el caso de los proyectos liderados por personas jurídicas, los resultados generales del estudio coinciden también en lo que respecta a los pares de lengua de mayor frecuencia de traducción, si bien, como era de esperar, la mayoría traduce a una lengua extranjera, en este caso, al inglés y francés a partir del español. Cabe destacar que en 13 de los 14 proyectos realizados por responsables extranjeros se traducen textos literarios.

Por último, en cuanto a los 19 proyectos encabezados por responsables residentes en regiones del norte y sur del país, se observa que los pares más frecuentes de traducción, al igual que en el caso de las personas jurídicas, son del «francés al español» (7) y del «inglés al español» (5), y en casi un 70\% de los casos se traducen textos literarios.

\section{DISCUSIÓN DE RESULTADOS}

La primera característica que quisiéramos destacar del concurso de apoyo a la traducción es el aumento considerable tanto del número de pro- yectos como de los fondos adjudicados para su financiamiento. Al consultar sobre las razones de este aumento a una gestora de proyectos del programa de internacionalización del libro del Consejo Nacional del Libro y la Lectura, nos señaló que el principal motivo era el énfasis que se le ha dado a este concurso dentro de la estrategia de internacionalización del sector editorial, lo cual, a su juicio, está relacionado con el incremento de las postulaciones recibidas y también con la calidad de las mismas. De hecho, en las bases del concurso se deja constancia expresa de que el Consejo podrá aumentar el presupuesto de cada convocatoria con la finalidad de financiar una mayor cantidad de proyectos elegibles. Este hecho refleja un interés gubernamental cada vez mayor por contribuir al fomento del libro y la lectura en nuestro país por medio de la traducción al español de obras escritas en lenguas extranjeras, y la traducción de obras escritas en español a lenguas extranjeras. De esta manera, la traducción se reconoce como un vehículo importante que permite fortalecer la industria editorial nacional y su internacionalización.

Por otra parte, el alto porcentaje de participación de personas naturales como responsables de los proyectos frente a personas jurídicas, en su mayoría editoriales, se debería a que 
la labor que realizan los traductores en Chile es un servicio altamente externalizado dado que el traductor «trabaja en forma independiente, ya sea de manera totalmente independiente o combinando esta modalidad con el trabajo en alguna organización» (Diéguez et al., 2015:16).

Entre las personas naturales responsables de los proyectos se cuentan en su mayoría traductores, escritores, poetas y personas con estudios de doctorado en diversas disciplinas. En menor medida han ejecutado proyectos profesores de educación superior, editores, periodistas e ilustradores. En lo que respecta a las personas jurídicas, se trata principalmente de pequeñas y microeditoriales independientes creadas entre los años 2006, las más antiguas, y 2014, las más recientes. No figuran entre las personas jurídicas editoriales de larga trayectoria ni grandes grupos editoriales del país o del extranjero. Podemos inferir entonces que la línea de apoyo a la traducción ha beneficiado principalmente a pequeñas y microeditoriales cuyo surgimiento en nuestro país en las últimas décadas es, a juicio de Fuentes et al. (2015), uno de los fenómenos más llamativos del campo editorial contemporáneo ${ }^{7}$. A juicio de estos autores, la emergencia de este tipo de editoriales se explica en parte por el acceso a las nuevas tecnologías de impresión, digitalización, digitación y policopiado.

En cuanto a la región de residencia de los responsables de los proyectos, las cifras generales reflejan el fenómeno de la centralización que caracteriza a nuestro país en lo que respecta a la actividad de traducción, tendencia que es más evidente aún al observar los datos de los proyectos realizados por personas jurídicas donde casi

El estudio de Fuentes et al. (2015) presenta una breve historia de la edición independiente en América Latina y Chile, y una descripción de la pequeña industria editorial en Chile y de su organización. la totalidad de ellas residen en la capital del país. Estos resultados coinciden con los obtenidos en el estudio de mercado de la traducción en Chile (2015) en el cual se concluye que, en materia de prestación de servicios profesionales de traducción, un alto porcentaje de los traductores que participaron en el estudio residen en la región Metropolitana (Diéguez et al., 2016: 4). No obstante lo anterior, los resultados del concurso muestran una tendencia incipiente a revertir esta situación, dado que, como ya se señaló, en el período de cinco años se ha pasado de una nula presencia de responsables residentes en regiones del sur y norte del país en el año 2013 a un porcentaje promedio de $28 \%$ en el período 2014 a 2017.

Digno de destacar es el hecho de que un porcentaje no menor de los proyectos es realizado por los propios autores de los textos originales. $\mathrm{Al}$ observar los datos correspondientes a los proyectos de autotraducción, comprobamos que ocho responsables son de nacionalidad chilena y seis son extranjeros, y que la gran mayoría, 11 de 14 proyectos, corresponden a géneros literarios como poesía, novela y ensayo. Este hecho corrobora la tendencia a la autotraducción en el ámbito de la traducción literaria, más que en el ámbito científico y de las ciencias sociales. Este sería un tema interesante de investigar en nuestro país con el fin de caracterizar esta práctica que en palabras de Tanqueiro (2002) no es «de dominio exclusivo de [...] sociedades plurilingües, sino que se produce en todas las partes del mundo y puede calificarse incluso de fenómeno natural [...]» (Cit. en Recuenco Peñalver, 2011:197). Entre otros aspectos, se podría investigar el nivel de bilingüismo, de estudios formales y de experiencia en traducción de los autotraductores.

La base de datos de los 90 proyectos presenta una gran variedad de autores de las obras a traducir ya que la mayor parte de ellos aparece solo 
218 una vez por proyecto, con la excepción de los poetas chilenos Alejandro Zambra (4 proyectos), Raúl Zurita ( 2 proyectos) y Pablo Jofré ( 2 proyectos); también figuran con dos proyectos la novelista chilena Lina Meruane y el poeta griego, Yannis Ritsos. Los autores extranjeros traducidos (63\%) superan a los autores chilenos representando a países como Francia (15 proyectos), Estados Unidos de Norteamérica (11 proyectos), Gran Bretaña (9 proyectos), Alemania (4 proyectos) e Italia y Grecia (3 proyectos cada uno). Con dos proyectos aparecen escritores de Suecia y con uno, autores de Suiza, la región china del Tíbet, Sudáfrica, Rusia, Perú, Brasil y Portugal. Por otra parte, más del $80 \%$ de los autores son contemporáneos y aproximadamente un 10\% del siglo XIX. También se presenta un autor del siglo XVIII, uno del siglo XVII, el maestro budista Sakia Pandita (1182-1251) y el poeta Horacio (65-8 a. C.). En el anexo se presentan los 90 proyectos del período con el año de recepción del proyecto, su responsable, el título de la obra a traducir y su autor o autora.

En lo que respecta a los pares de lengua de mayor frecuencia de traducción, en los resultados de este estudio se observan algunas similitudes y diferencias con los estudios de mercado de la traducción en Chile realizados los años 2011 y 2015. En efecto, existe coincidencia en que las lenguas de mayor frecuencia de traducción en Chile son los pares del «inglés al español» y del «español al inglés» que en este estudio se sitúan en el primer y tercer lugar, respectivamente. En efecto, estos dos pares de lengua de traducción son los más frecuentes en los estudios de mercado realizados en nuestro país (Diéguez et al., 2014: 77 y Diéguez et al., 2016: 9).

Las diferencias se manifiestan en el par de lenguas del «español al francés» que en este estudio ocupa el segundo lugar con 17 proyectos, luego del par de lenguas del «inglés al español» que es el más frecuente con 25 proyectos. El par de lenguas del «español al francés» supera al par del «español al inglés» que ocupa el segundo lugar en los estudios de mercado citados anteriormente. Otra diferencia es la presencia de los pares de lengua del «español al italiano» y del «italiano al español» en cuarto y quinto lugar de frecuencia; de esta manera, el italiano supera a lenguas como el alemán y el portugués, idiomas cuya frecuencia de traducción se ubica luego del inglés y el francés en los estudios de mercado 2011 y 2015.

Cabe señalar que entre los pares de lengua de los proyectos estudiados no figuran traducciones desde y hacia lenguas de pueblos originarios como el mapudungun, lengua del pueblo mapu$\mathrm{che}^{8}$. Este hecho demuestra que en los primeros 5 años de implementación de la línea de apoyo a la traducción no se ha cumplido con la medida señalada por la PNLL de traducir a lenguas originarias obras patrimoniales de los pueblos indígenas lo cual permitiría fomentar la creación y el emprendimiento editorial de los pueblos indígenas (Política Nacional del Libro y la Lectura, 2015: 36). Por ello, se debe optimizar, como señalamos más adelante en las conclusiones, la política de difusión del concurso, en especial en las regiones del país donde habitan principalmente los miembros de los pueblos originarios ${ }^{9}$.

En cuanto al formato en que se presentan las traducciones, se constata una bajísima presencia del formato digital, frente al formato impreso.

8 La Ley Indígena reconoce la existencia de nueve pueblos originarios en el país, cuatro de ellos con vitalidad lingüística (mapuche, aymara, rapa nui y quechua) y mandata al Ministerio de Educación a promover la enseñanza de las lenguas y culturas de los pueblos originarios en el sistema escolar (Ministerio de Educación, 2020).

9 En consulta con dos investigadoras de la cultura y lengua mapuche, se nos ha señalado que, si bien la traducción desde el mapudungun al español y viceversa es una práctica habitual en nuestro país, sería muy interesante estudiar a futuro las principales fuentes de financiamiento que reciben dichas iniciativas. 
Llama la atención que a pesar de que las bases del concurso permiten el formato digital, los responsables de los proyectos y las editoriales que publican las traducciones manifiestan una clara preferencia por el formato tradicional impreso. Esta tendencia coincide con los resultados del estudio de Fuentes et al. (2015: 31) quienes concluyen que las pequeñas y microeditoriales independientes se decantan en un elevado porcentaje (97\%) por el formato impreso. Un tema interesante de investigar a futuro serían las políticas y criterios en cuanto al tipo de formato, impreso o digital, que adoptan las editoriales al publicar traducciones en nuestro país.

Por otra parte, la alta presencia de textos literarios, entre los que se destacan poesía, novela y ensayo, frente a los no literarios responde en gran parte a los objetivos que se ha planteado el Fondo y que dicen relación, como ya señalamos, con el apoyo a la creación literaria y a la difusión de la actividad literaria. Digno de destacar es el elevado porcentaje de la poesía dentro de los textos literarios que se traducen, tendencia que sería interesante seguir investigando en las convocatorias al concurso del Fondo a partir del año 2018. También sería relevante comparar estos resultados con estudios similares realizados en otros países con el fin de describir similitudes y diferencias en cuanto a los géneros literarios que más se traducen con apoyo de fondos estatales ${ }^{10}$.

Cabe destacar que las bases del concurso ofrecen la posibilidad de traducir obras no literarias, dado que, como ya señalamos, solo se exige que los textos originales sean de ficción o no ficción y que pertenezcan a cualquier tipo de texto. Este

\footnotetext{
Io Pablo Núñez (2017) realiza una investigación sobre las ayudas del Ministerio de Cultura de España a la traducción de obras españolas en lenguas extranjeras en el período 2006-2011 y concluye que se presenta una clara tendencia hacia la traducción de textos narrativos.
}

hecho explicaría que alrededor de un cuarto de los textos originales corresponden a monografías sobre variadas temáticas relacionadas con aspectos culturales y de las ciencias sociales, como se puede observar en los resultados. Digno de mencionar es el hecho que los proyectos realizados por personas jurídicas presentan una frecuencia levemente mayor de textos literarios ya que la traducción de géneros literarios (14) supera a la de los géneros no literarios (9). En el caso de los responsables extranjeros y los que residen en regiones del norte y sur del país, se observa una marcada preferencia por traducir géneros literarios.

\section{CONCLUSIONES}

En este estudio se ha caracterizado el proceso de institucionalización de la disciplina y de la práctica de la traducción, se han presentado las principales características de los 90 proyectos de traducción financiados en el período 2013 a 2017 por la línea de apoyo a la traducción y, en esta última sección, se propondrán medidas para continuar consolidando la institucionalización de la traducción en nuestro país.

En lo que respecta al proceso de institucionalización de la traducción, podemos afirmar que se ha avanzado en las últimas décadas en los ámbitos académicos de investigación y formación profesional, y en los aspectos gremiales, jurídicos y normativos, amén de la práctica de la traducción, si bien aún queda mucho por hacer. En efecto, en lo que respecta a la investigación en traducción, constatamos a la fecha la ausencia de la etiqueta «traducción» en la lista de clasificación por disciplinas científicas y tecnológicas para la postulación a proyectos FONDECYT, situación similar a la que ocurre en Argentina, donde esta etiqueta también está ausente en la lista de dis- 
220

ciplinas del Consejo Nacional de Investigaciones Científicas y Técnicas, CONICET, (Arrizabalaga, 2013: 43-44). En ambos países, los proyectos de traducción se deben adscribir a áreas como la literatura, lingüística, filología o semiótica.

\section{¿Cómo optimizar el proceso de institucionalización de la traducción?}

Frente a lo planteado anteriormente, consideramos pertinente proponer algunas medidas que permitan contribuir a la optimizar la institucionalización de la traducción en nuestro país:

I. Aprovechar la oportunidad que ofrece el Fondo de Fomento del Libro y la Lectura para continuar desarrollando proyectos de traducción que contribuyan a fortalecer la industria editorial nacional y su internacionalización.

2. Optimizar la política de difusión del concurso de apoyo a la traducción en todo el país, en especial en regiones, adoptando diferentes acciones como publicaciones, conferencias, talleres, ponencias, mesas redondas, entre otras.

3. Continuar con la participación de representantes del COTICH en la Comisión de trabajo sobre Internacionalización del libro y la lectura en el marco de la Política Nacional del Libro y la Lectura (2015).

4. Incentivar la formación de posgrado a nivel de magíster y doctorado de los estudiantes de traducción que se forman a nivel de licenciatura en nuestro país.

5. Fomentar la investigación en diferentes áreas de la traducción como, por ejemplo, estudios de mercado, autotraducción, políticas editoriales en materia de traducción y traducción de lenguas de pueblos originarios, entre muchas otras.
6. Incentivar el uso del formato digital de las traducciones con el fin de aumentar la divulgación de las obras traducidas.

Reiteramos que se trata de un primer estudio exploratorio descriptivo cuyos resultados se deben complementar a futuro con investigaciones en áreas específicas como la investigación, la docencia y la vinculación con el medio en el ámbito de la traducción en Chile. Estos resultados permitirán nutrir y a la vez nutrirse de la práctica de la traducción y de sus dimensiones académicas, gremiales, jurídicas y normativas. Esperamos que estos datos sean de utilidad tanto para investigadores, profesores y estudiantes de traducción, como también para traductores profesionales quienes podrán conocer y aprovechar las oportunidades que ofrece este tipo de políticas públicas con miras a fomentar la traducción en nuestro país.

A la luz de los resultados de este estudio, podemos afirmar que la creación en 2013 de la línea de apoyo a la traducción del Fondo de Fomento del Libro y la Lectura y la publicación al año siguiente de la Política Nacional de la Lectura y el Libro 2015-2020 por parte del Consejo Nacional de la Cultura y las Artes constituyen dos iniciativas gubernamentales que han contribuido en los últimos años a consolidar la institucionalización de la traducción en nuestro país a través del concurso de fondos de apoyo para la traducción, a lo cual sumamos las actividades de difusión que deben realizar los responsables en establecimientos de educación pública y sus comunidades. Reiteramos también que la participación de los representantes del COTICH en las mesas de trabajo contribuyó y seguirá contribuyendo, junto a otros numerosos participantes, a materializar esta política gubernamental que propone incentivar la lectura con el fin de hacer de nuestros compatriotas personas más informadas, críticas, reflexivas y participativas. 


\section{REFERENCIAS BIBLIOGRÁFICAS}

ARAYA, Cristian (2013): El enfoque por competencias en la formación de traductores: análisis de necesidades a nivel de posgrado en Chile, Tesis Universidad de Ginebra, Ginebra: Universidad de Ginebra. <https://archive-ouverte.unige.ch/unige:33083>

Arrizabalaga, María Inés (2013): «Four Models and a Challenge. Past, present, and future of translator training programs in Argentina», Connexions, International Professional Communication Journal, 1/1, 39-45. <https://ri.conicet.gov.ar/handle/11336/23324>

Basaure, Rosa y Marcela Contreras (2019): «Movimientos feministas y enfoques de género: integración del debate en las líneas curriculares para la formación de traductores en Chile», Mutatis Mutandis, 12 (1), 156-181. <http://aprendeenlinea.udea. edu.co/revistas/index.php/mutatismutandis/article/view/33741>

BRodsky, Ricardo (2013): «La mesa y la política del libro: una experiencia inacabada», Comunicación y Medios, 27, 163-169. <https://comunicacionymedios. uchile.cl/index.php/RCM/article/view/24928>

Cabrera, Ileana, Patricia Hörmann, Emilio López y Juan Carlos Palazuelos (1991): Investigación en traducción. Planteamientos y perspectivas, Santiago, Chile: Pontificia Universidad Católica de Chile.

CABrera, Ileana (1995): «Apuntes sobre el desarrollo de la traducción en Chile», en Actas del IV Congreso Internacional de "El Español de América", Tomo II. Santiago, Chile: Instituto de Letras, Pontificia Universidad Católica de Chile, 1317-1323.

Colegio de Traductores e Intérpretes de Chile (2019): «Quiénes somos». <http://www.cotich.cl/ quienes-somos/> [consulta: $31-$ III-2019].

Consejo Nacional del Libro y la Lectura. Ministerio de las Culturas, las Artes y el Patrimonio (2019): <www. cultura.gob.cl/libro-y-lectura/cnll/>, [consulta: 31-III- 2019].

Cronin, Michael (2013): «Translation and globalization», en Carmen Millán y Francesca Bartrina (eds.), The Routledge Handbook of Translation Studies, Londres: Routledge, 491-502.

Delgado, Gonzalo (2017): Éxito en la carrera laboral en traductores según su trayectoria laboral, Tesis Universidad de Concepción, Santiago, Chile: Universidad de Concepción. <http://repositorio.udec.cl/bitstream/handle/11594/2615/Te sis_Exito_en_la_carrera_laboral_en_traductores. pdf? sequence $=1 \&$ isAllowed $=y>$

DiÉGuez, María Isabel, Rosa María Lazo y Camilo QuezADA (2014): «Estudio de mercado de la traducción en Argentina, Chile y España: perfil académico y profesional de los traductores», Onomázein, 30/2, 70-89. <http://onomazein.letras.uc.cl/Articulos/ N30/30_6_Dieguez_FINAL.pdf>

Diéguez, María Isabel, Rosa María Lazo y Camilo Quezada (2015): «Perfil del teletraductor en Argentina, Chile y España: las TIC aplicadas a la práctica profesional», Onomázein, 31/1, 1-19. < http://onomazein.letras.uc.cl/Articulos/N31/31_2_Dieguez. pdf>

Diéguez, María Isabel, Rosa María Lazo y Carles Tebé (2016): Informe final del estudio de mercado de la traducción en Chile (2015), Documento interno, Santiago, Chile: Programa de Traducción, Facultad de Letras, Pontificia Universidad Católica de Chile. <http://programas.letras.uc.cl/ index.php/actividades-de investigacion?view=article\&id=61:estudio-de-mercado-de-la-traduccion-en-chile-2015>

FuENTES, Lorena et al. (2015): La edición independiente en Chile. Estudio e historia de la pequeña industria (2009-2014), Santiago, Chile: Cooperativa de Editores de la Furia. <https://www.uchile.cl/portal/ extension-y-cultura/vicerrectoria-de-extensiony-comunicaciones/observatorio-del-libro-y-lalectura/estudios/89768/el-mercado-del-librochileno>

Instituto NACional de NoRMALIZACión (2008): NCh 3124. Of2008 Servicios de traducción - Requisitos para la prestación de servicios, Santiago, Chile: Instituto Nacional de Normalización.

Instituto NACional de Normalización (2008): NCh 3159. Of2008 Documentación - Presentación de traducciones, Santiago, Chile: Instituto Nacional de Normalización.

LAMBERT, José (2013): «The institutionalization of the discipline», en Carmen MILLÁN y Francesca BAR- 
TRINA (eds.), The Routledge Handbook of Translation Studies, Londres: Routledge, 7-27.

Micheli, Paula (2013): La subcompetencia instrumental profesional dentro del currículum de traducción en Chile. Estado del arte y propuesta curricular, Tesis Universidad Autónoma de Barcelona: Universidad de Barcelona. <https://www.academia. edu/33824801/Subcompetencia_instrumental_ profesional_en_Chile_estado_del_arte_y_propuesta_curricular>

Ministerio de Educación (2017): Ley de Propiedad Intelectual (1970/2017), Santiago, Chile: Gobierno de Chile. «https://www.leychile.cl/ Navegar?idNorma $=28933>$ [consulta: 31-III2019].

Ministerio de Educación (2020): Lenguas originarias, Santiago, Chile: Gobierno de Chile. <http://peib. mineduc.cl/lenguas-originarias-2/> [consulta: 10-VI-2020].

Ministerio de Justicia (2019): Código de Procedimiento Civil (1902/2019), Santiago, Chile: Gobierno de Chile. <https://www.leychile.cl/ Navegar?idNorma $=22740>$ [consulta: 31-III2019].

Ministerio de las Culturas, las Artes y el Patrimonio. Consejo Nacional de la Cultura, las Artes y el PatRIMONIO (2015): Política Nacional de la Lectura y el Libro 2015-2020, Santiago, Chile. <https://www. cultura.gob.cl/politicas-culturales/lectura-libro/> [consulta: 28-III-2019].

Ministerio de las Culturas, las Artes y el PatrimoNio (2019): Bases de concurso público del Fondo Nacional de Fomento del Libro y La lectura, programa de apoyo a la traducción, convocatoria 2019. <https://www.fondosdecultura.cl/wp-content/ uploads/2019/03/libro-bases-traduccion-2019. pdf $>$, [consulta: 31-III-2019].

Pablo NúÑEz, Luis (2017): «Las ayudas estatales a la traducción de obras españolas en lenguas extranjeras: balance de los años 2006-2011», Itinerarios, 25, 167-191. <http://hdl.handle.net/10481/47308>

PAYÀs, Gertrudis (2007): Biblioteca chilena de traductores (1820-1924). Ordenada por José Toribio Medina. Segunda edición, corregida y aumentada. Con estudio preliminar de Gertrudis Payàs, Centro de Investiga- ciones Diego Barros Arana. Santiago, Chile: Ediciones de la Dirección de Bibliotecas, Archivos y Museos.

Recuenco Peñalver, María (2011): «Más allá de la traducción: la autotraducción», Trans, 15, 193-208. $<$ http://www.trans.uma.es/pdf/Trans_15/193-208. $\mathrm{pdf}>$ 
ANEXO

Nómina de proyectos de traducción del Fondo Nacional de Fomento del Libro y la Lectura (2013-2017)

\begin{tabular}{|c|c|c|c|}
\hline $\begin{array}{l}\text { FECHA DE } \\
\text { RECEPCIÓN }\end{array}$ & RESPONSABLE & OBRA A TRADUCIR & AUTOR \\
\hline 2013 & Lina Meruane Boza & Sangre en el ojo & Lina Meruane Boza \\
\hline 2013 & Nadine Paule Barbara Dejong & La ley de Snell & Leonardo Sanhueza \\
\hline 2013 & Nicolás Hernán Poblete Pardo & Cardumen & Nicolás Hernán Poblete \\
\hline 2013 & $\begin{array}{l}\text { Christian Enrique Formoso } \\
\text { Bavich }\end{array}$ & $\begin{array}{l}\text { El cementerio más hermoso } \\
\text { de Chile }\end{array}$ & Christian Enrique Formoso \\
\hline 2013 & $\begin{array}{l}\text { Andrea Mariana Jeftanovic } \\
\text { Avdaloff }\end{array}$ & Geografía de la lengua & Andrea Jeftanovic \\
\hline 2013 & Eduardo Ruiz-Tagle Eyzaguirre & $\begin{array}{l}\text { Takona Tatu. Tatuajes de } \\
\text { Isla de Pascua }\end{array}$ & Ana María Arredondo \\
\hline 2013 & Lina Meruane Boza & Viajes virales & Lina Meruane \\
\hline 2013 & Pedro Antonio Araya Riquelme & Las ciudades de agua & Raúl Zurita \\
\hline 2013 & Pedro Antonio Araya Riquelme & Mudanza & Alejandro Zambra \\
\hline 2013 & $\begin{array}{l}\text { Diego Patricio Muñoz Valen- } \\
\text { zuela }\end{array}$ & Flores para un Cyborg & Diego Muñoz \\
\hline 2014 & Patricio Iván García Maldonado & Escrito & Andrés Morales \\
\hline 2014 & Pedro Antonio Araya Riquelme & Alto Volta & Yanko González Cangas \\
\hline 2014 & Patricio Iván García Maldonado & Luz rabiosa & Rafael Rubio \\
\hline 2014 & Patricio Iván García Maldonado & $\begin{array}{l}\text { Canto a su amor desapa- } \\
\text { recido }\end{array}$ & Raúl Zurita \\
\hline 2014 & $\begin{array}{l}\text { Fresia Barrientos Morales EIRL } \\
\text { Nativa Ediciones }\end{array}$ & Pueblos originarios de Chile & Fresia Barrientos Morales \\
\hline 2014 & $\begin{array}{l}\text { Gilberto Villarroel Escobar } \\
\text { Producciones Audiovisuales y } \\
\text { Editoriales EIRL }\end{array}$ & Lovecraft vive en Chile & Gilberto Villarroel \\
\hline 2014 & José Pablo Jofré Jofré & Abecedario (al griego) & José Pablo Jofré Jofré \\
\hline 2014 & Editorial Hueders Ltda. & Mis documentos & Alejandro Zambra \\
\hline 2014 & Editorial Hueders Ltda. & Facsímil & Alejandro Zambra \\
\hline 2014 & Editorial Hueders Ltda. & No leer & Alejandro Zambra \\
\hline 2014 & Emilio Gordillo Lizana & Croma & Emilio Gordillo Lizana \\
\hline
\end{tabular}




\begin{tabular}{|c|c|c|c|}
\hline 2014 & Antonia Tatiana Torres Agüero & Umzug & Antonia Torres \\
\hline 2015 & Franck Gaudichaud Miron & $\begin{array}{l}\text { Chili 1970-1973. Mille jours } \\
\text { qui ébranlèrent le monde }\end{array}$ & Franck Gaudichaud \\
\hline 2015 & $\begin{array}{l}\text { Natalia Alejandra Figueroa } \\
\text { Gallardo }\end{array}$ & 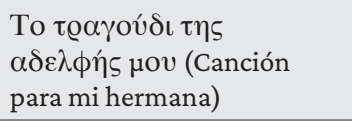 & Yannis Ritsos \\
\hline 2015 & $\begin{array}{l}\text { Ediciones e Impresiones Metales } \\
\text { Pesados Ltda. }\end{array}$ & $\begin{array}{l}\text { Tecnologías de la crítica. } \\
\text { Entre Walter Benjamin y } \\
\text { Gilles Deleuze }\end{array}$ & Willy Thayer Morel \\
\hline 2015 & $\begin{array}{l}\text { Ediciones e Impresiones Metales } \\
\text { Pesados Ltda. }\end{array}$ & $\begin{array}{l}\text { Making Art Panamerican. } \\
\text { Cultural Policy and the } \\
\text { Cold War }\end{array}$ & Claire F. Fox \\
\hline 2015 & $\begin{array}{l}\text { Ediciones e Impresiones Metales } \\
\text { Pesados Ltda. }\end{array}$ & Die Kraft der Kunst & Christoph Menke \\
\hline 2015 & $\begin{array}{l}\text { Ediciones e Impresiones Metales } \\
\text { Pesados Ltda. }\end{array}$ & $\begin{array}{l}\text { À coups de points. La } \\
\text { ponctuation comme } \\
\text { expérience }\end{array}$ & Peter Szendy \\
\hline 2015 & $\begin{array}{l}\text { Ediciones e Impresiones Metales } \\
\text { Pesados Ltda. }\end{array}$ & $\begin{array}{l}\text { Aliénation: Antonin Artaud. } \\
\text { Les généalogies hybrides }\end{array}$ & Serge Margel \\
\hline 2015 & Zilin Cui & $\begin{array}{l}\text { Neruda: Memoria crepi- } \\
\text { tante }\end{array}$ & Virginia Vidal \\
\hline 2015 & Camila Iver Morales & $\begin{array}{l}\text { Rongo. La historia oculta de } \\
\text { Isla de Pascua }\end{array}$ & Patricia Stambuk \\
\hline 2015 & Carolina Yazigi Waissbluth & $\begin{array}{l}\text { Adventures in the Anthro- } \\
\text { pocene: A Journey to the } \\
\text { Heart of the Planet we } \\
\text { Made }\end{array}$ & Gaia Vince \\
\hline 2015 & Tomás Esteban Cohen Herrera & The Cloud Corporation & Timothy Donnelly \\
\hline 2015 & La Pollera Ediciones Ltda. & Mémoires d'un amnésique & Erik Satie \\
\hline
\end{tabular}

2015 Rodrigo Andrés Olavarría Lavín

Benito Cereno; Narrative of Voyages in the Northern and Southern Hemisphere

\begin{tabular}{llll}
\hline 2015 & Rodrigo Andrés Olavarría Lavín & Not Me & Eileen Myles \\
\hline 2015 & Tomás Esteban Cohen Herrera & El Tesoro de Aforismos & Sakya Pandita \\
\hline
\end{tabular}

Amasa Delano y Herman Melville 


\begin{tabular}{|c|c|c|c|}
\hline 2016 & Cecilia Alejandra Bettoni Piddo & $\begin{array}{l}\text { The Remembrance of } \\
\text { Things Past. On Aby M. } \\
\text { Warburg and Walter Ben- } \\
\text { jamin }\end{array}$ & Matthew Rampley \\
\hline 2016 & La Pollera Ediciones Ltda. & How I Found Livingstone & Henry Stanley \\
\hline 2016 & Camila Iver Morales & $\begin{array}{l}\text { How The World Changed } \\
\text { Social Media }\end{array}$ & $\begin{array}{l}\text { Daniel Miller, Elisabetta } \\
\text { Costa, Nell Haynes, Tom } \\
\text { McDonald, Razvan Nicolescu, } \\
\text { Jolynna Sinanann, Juliano } \\
\text { Spyer, Shriram Venkatraman, } \\
\text { Xinyuan Wang }\end{array}$ \\
\hline 2016 & Pablo Jofré Jofré & Abecedario (trilingüe) & Pablo Jofré Jofré \\
\hline 2016 & $\begin{array}{l}\text { Arquitectura y Diseño Francisca } \\
\text { Alejandra Muñoz Méndez EIRL }\end{array}$ & Beyond Urbanism & Jeannette Sordi \\
\hline 2016 & Editorial Doble Ciencia Ltda. & $\begin{array}{l}\text { Faire la paix avec l'animal } \\
\text { (Hacer las paces con el } \\
\text { animal) }\end{array}$ & Dominique Lestel \\
\hline 2016 & $\begin{array}{l}\text { Cristian Rodrigo Medina Mal- } \\
\text { donado }\end{array}$ & Mi lai & Carmen Berenguer \\
\hline 2016 & $\begin{array}{l}\text { Enrique Andrés Winter Sepúl- } \\
\text { veda }\end{array}$ & Antología poética & $\begin{array}{l}\text { Enrique Andrés Winter } \\
\text { Sepúlveda }\end{array}$ \\
\hline 2016 & $\begin{array}{l}\text { Cristóbal Enrique Joannon } \\
\text { Ljubetic }\end{array}$ & Aforismos & William Temple \\
\hline 2016 & $\begin{array}{l}\text { Luz María de Lourdes Astudillo } \\
\text { Ugalde }\end{array}$ & $\begin{array}{l}\text { Complete Poems and Let- } \\
\text { ters }\end{array}$ & Wilfred Owen \\
\hline 2016 & Fundación Nicomedes Guzmán & $\begin{array}{l}\text { Nicomedes Guzmán: Pro- } \\
\text { letarian Author in Chile’s } \\
\text { Literary Generation of } 1938\end{array}$ & Lon Pearson \\
\hline 2016 & Patricio Iván García Maldonado & Sueños de luna azul & Elicura Chihuailaf \\
\hline 2016 & $\begin{array}{l}\text { Aude Marie Pierre Hélène Gene- } \\
\text { viève Argouse }\end{array}$ & $\begin{array}{l}\text { El orden gañán. Historia so- } \\
\text { cial de la Policía. Valparaíso } \\
1896-1920\end{array}$ & Vania Cárdenas Muñoz \\
\hline
\end{tabular}




\begin{tabular}{|c|c|c|c|}
\hline 2016 & Juan Carlos Toro Lecaros & $\begin{array}{l}\text { Die Hamletmaschine; } \\
\text { Anatomie Titus Fall of } \\
\text { Rome Ein Shakespearekom- } \\
\text { mentar; Verkommenes Ufer } \\
\text { Medeamaterial Landschaft } \\
\text { mit Argonauten; Bildbe- } \\
\text { schreibung; Herakles } 5\end{array}$ & Heiner Müller \\
\hline 2016 & Patricio Iván García Maldonado & En un abrir y cerrar de ojos & Oscar Hahn \\
\hline 2016 & Mónica Carla Ruiz Fuentes & Job-boj & Jorge Guzmán \\
\hline 2016 & Carlos Soto Román & $\begin{array}{l}\text { Space Between these Lines } \\
\text { Not Dedicated }\end{array}$ & Frank Sherlock \\
\hline 2016 & $\begin{array}{l}\text { Juan Cristóbal Romero Bucci- } \\
\text { cardi }\end{array}$ & $\begin{array}{l}\text { Libro Primero de las Epís- } \\
\text { tolas }\end{array}$ & Horacio \\
\hline 2016 & Cristian Rodrigo Olivos Bravo & Le livre de Monelle & Marcel Schwob \\
\hline 2016 & Magdalena Antosz & $\begin{array}{l}\text { La extinción de los } \\
\text { coleópteros }\end{array}$ & Diego Vargas Gaete \\
\hline 2016 & Patricio Iván García Maldonado & Porquoi Colombe? & Nicole Barriere \\
\hline 2016 & Omar Pérez Santiago & No-Muerto & Clemens Altgard \\
\hline 2016 & $\begin{array}{l}\text { Galo Geraldo Ghigliotto Ghi- } \\
\text { gliotto }\end{array}$ & $\begin{array}{l}\text { Il faut beaucoup aimer les } \\
\text { hommes }\end{array}$ & Marie Darrieussecq \\
\hline 2016 & Ambos Editores & Jérémie! Jérémie! & Dominique Fernández \\
\hline 2017 & Nicolás Alejandro Trujillo Osorio & $\begin{array}{l}\text { Die Begriffsform im myti- } \\
\text { schen Denken }\end{array}$ & Enrst Cassirer \\
\hline 2017 & Cecilia Alejandra Bettoni Piddo & La vie des formes & Henri Focillon \\
\hline 2017 & Camila Iver Morales & $\begin{array}{l}\text { The Autobiography of a } \\
\text { Seaman }\end{array}$ & Lord Cochrane \\
\hline 2017 & $\begin{array}{l}\text { Galo Geraldo Ghigliotto Ghi- } \\
\text { gliotto }\end{array}$ & Panorámica de Andrade & Mário de Andrade \\
\hline 2017 & Editorial Librosdementira Ltda. & $\begin{array}{l}\text { The Crime of Captain Ga- } \\
\text { hagan; The Unmentionable } \\
\text { Man; Ring of Lovers }\end{array}$ & Gilbert Keith Chesterton \\
\hline 2017 & $\begin{array}{l}\text { Macarena Francisca García } \\
\text { Moggia }\end{array}$ & Neve, cane, piede & Claudio Morandini \\
\hline 2017 & Cecilia Alejandra Bettoni Piddo & $\begin{array}{l}\text { Les cadres sociaux de la } \\
\text { mémoir }\end{array}$ & Maurice Halbwachs \\
\hline
\end{tabular}




\begin{tabular}{|c|c|c|c|}
\hline 2017 & $\begin{array}{l}\text { Ediciones y Publicaciones } \\
\text { Verónica del Carmen Jiménez } \\
\text { Dotte EIRL }\end{array}$ & Ace and Other Poems & Tom Raworth \\
\hline 2017 & $\begin{array}{l}\text { Natalia Alejandra Figueroa } \\
\text { Gallardo }\end{array}$ & Diarios de exilio & Yannis Ritsos \\
\hline 2017 & Raúl Alejandro Rodríguez Freire & Derrida y la literatura & Jacques Derrida \\
\hline 2017 & María Isabel Diéguez Morales & Fertigkeit Übersetzen & Christiane Nord \\
\hline 2017 & $\begin{array}{l}\text { Galo Geraldo Ghigliotto Ghi- } \\
\text { gliotto }\end{array}$ & $\begin{array}{l}\text { Mémoires d'un homme } \\
\text { singulier }\end{array}$ & Emmanuel Bove \\
\hline 2017 & Lorena Adriana Fuentes Reyes & Los condenados & Daniel Alarcón \\
\hline 2017 & Árbol Bricolage S.A. & Antología de poemas & John Berryman \\
\hline 2017 & Pablo Javier Abufom Silva & $\begin{array}{l}\text { Black Flame. The Revolutio- } \\
\text { nary Class Politics of Anar- } \\
\text { chism and Syndicalism }\end{array}$ & Lucien van der Walt \\
\hline 2017 & Amarí Peliowski Dobbs & $\begin{array}{l}\text { Voyaging: Southward from } \\
\text { the Strait of Magellan }\end{array}$ & Rockwell Kent \\
\hline 2017 & Edicola SpA & Un tango per Victor & Lorenzo Mazzoni \\
\hline 2017 & Cristian Rodrigo Olivos Bravo & Le vin est tiré & Robert Desnos \\
\hline 2017 & María José Navia Torelli & Battleborn & Claire Vaye Watkins \\
\hline 2017 & Cristina Varas Largo & $\begin{array}{l}\text { Клоп (La chinche); Баня } \\
\text { (el baño) }\end{array}$ & Vladímir Maiakovski \\
\hline 2017 & $\begin{array}{l}\text { Ediciones e Impresiones Metales } \\
\text { Pesados Ltda. }\end{array}$ & Gesellschaft & Dirk Baecker \\
\hline 2017 & $\begin{array}{l}\text { Ediciones Bastante María del } \\
\text { Rosario Garrido EIRL }\end{array}$ & On the Pleasure of Hating & William Hazlitt \\
\hline 2017 & $\begin{array}{l}\text { Andrea Patricia Quezada Rich- } \\
\text { ards }\end{array}$ & Dramaturgia sonora & Valentina Valentini \\
\hline 2017 & $\begin{array}{l}\text { Soc. Gloria Elgueta y Claudia } \\
\text { Marchant Ltda. }\end{array}$ & $\begin{array}{l}\text { Là où se termine la terre. } \\
\text { Chili 1948-1970. }\end{array}$ & Désirée y Alain Frappier \\
\hline 2017 & Julio Iván Carrasco Ruiz & $\begin{array}{l}\text { Sesenta poemas de Guillau- } \\
\text { me Apollinaire }\end{array}$ & Guillaume Apollinaire \\
\hline
\end{tabular}




\begin{tabular}{llll}
\hline 2017 & Juan Manuel Silva Barandica & $\begin{array}{l}\text { Amistad, amor y matri- } \\
\text { monio }\end{array}$ & Henry David Thoreau \\
\hline 2017 & $\begin{array}{l}\text { Ediciones e Impresiones Metales } \\
\text { Pesados Ltda. }\end{array}$ & $\begin{array}{l}\text { Paisajes, Proyecciones, } \\
\text { Perversiones: Perspectivas } \\
\text { en Teoría Queer }\end{array}$ & Jill H. Casid \\
\hline 2017 & $\begin{array}{l}\text { Ediciones y Publicaciones } \\
\text { Verónica del Carmen Jiménez } \\
\text { Dotte EIRL }\end{array}$ & $\begin{array}{l}\text { 35 Sonnets and English } \\
\text { Poems }\end{array}$ & Fernando Pessoa \\
\hline 2017 & Juan Carlos Villavicencio Ortega & Antología poética & Sara Teasdale \\
\hline
\end{tabular}

